\title{
Proximity Templates for Modeling of Skin and Proximity Effects on Packages and High Frequency Interconnect .
}

\author{
Luca Daniel \\ Massachusetts Instit. of Tech. \\ dluca@mit.edu
}

\author{
Alberto \\ Sangiovanni-Vincentelli \\ Univ. of California, Berkeley \\ alberto@eecs.berkeley.edu
}

\author{
Jacob White \\ Massachusetts Instit. of Tech. \\ white@mit.edu
}

\begin{abstract}
Modeling the exponentially varying current distributions in conductor interiors associated with high frequency interconnect behavior causes a rapid increase in the computation time and memory required even by recently developed fast electromagnetic analysis programs. In this paper we describe a procedure to generate numerically a set of basis functions which efficiently represent conductor current variation, and thus improving solver efficiency. The method is based on solving a sequence of template problems, and is easily generalized to arbitrary conductor cross-sections. Results are presented to demonstrate that the numerically computed basis functions are seven to twenty times more efficient than the commonly used piece-wise constant basis functions.
\end{abstract}

Categories \& Subject Descriptors: B.7.2 Simulation, B.8.2 Performance Analysis \& Design Aids, I.6 Simulation \& Modeling.

General Terms: Algorithms, Performance.

Keywords: Skin Effect, Proximity Effect, Parasitic extraction, Interconnect analysis.

\section{INTRODUCTION}

The new generation of fast electromagnetic analysis programs, based on accelerated integral equation methods, has reduced from days to minutes the time required to analyze thousands of simultaneously interacting conductors $[1,2,3,4,5]$. As good as these fast solvers are, they are either inappropriate for, or are very inefficient at, analyzing interconnect exhibiting high frequency effects. With processor clock speeds now exceeding two gigahertz and harmonics exceeding twenty gigahertz, it is no longer possible to ignore these high frequency effects.

The high frequency effects that are most troublesome for fast solvers are skin and proximity effects. Nevertheless such phenomena can significantly affect interconnect performance and should not be neglected, in particular when either wire width or thickness are equal to, or larger than two "skin-depths." As clock frequencies rise, skin effects have become more significant in printed circuit

\footnotetext{
${ }^{*}$ This work was supported by the MARCO Interconnect Focus Center and the Semiconductor Research Corporation.
}

boards (PCB) and IC packages, and such effects are even becoming important for on-chip interconnect. When one considers that the skin depth in aluminum drops below a micron at approximately ten gigahertz, it is not surprising that skin effects are seen in IC's with multi-gigahertz clocks.

Skin and proximity effects are troublesome for present fast solver because they generate an exponentially varying current distribution inside each conductor. Trying to represent that current variation using the piece-wise constant $[6,7]$ or piece-wise linear basis functions commonly available in fast solvers [2] requires a large number of unknowns. Since the computation time for fast solvers is supposed to increase only linearly with the total number of basis functions used in the problem, it may seem that the increase in unknowns to represent current variation is not that problematic. However, when many basis functions are used to represent the current variation in a cross-section of a conductor, those basis functions densely interact in a way that can not be reduced by the algorithms used in most fast solvers. For this reason, the computation time for modeling high frequency effects increases with the square of the number of unknowns required to model the current variation within conductors even for fast solvers.

The rapid increase in fast solver computation time associated with modeling high frequency effects has focused research efforts on finding methods to either avoid representing currents in conductor interiors $[8,9,10,11,12,13]$, or to generate specialized basis functions which more easily capture the exponential variation of the conductor current $[14,15]$. In this paper we demonstrate that it is possible to generate numerically a set of basis functions which efficiently represent conductor current variation. Our method is based on solving a sequence of simple "template" problems for the typical geometries associated with a given interconnect technology. These template problem solutions are then used as replacement for the piece-wise constant basis functions in an integral equation method based on the Galerkin discretization [16] of the Mixed-Potential Integral Equation (MPIE) $[6,17,14]$. As our results will demonstrate, the numerically computed basis functions require 7 to 20 times fewer unknowns than piece-wise constant basis functions. It should be noted that similar performance was achieved by generating basis functions using 2-D conduction modes [14, 15], but unlike the conduction mode approach, the template approach is easily extended to general shape cross-sections (e.g. trapezoidal).

In the next section, we summarize the integral equation method based on the Galerkin discretization of the Mixed-Potential Integral Equation (MPIE). In Section 3 we describe the steps for the precomputation of our template basis functions. In Section 4 we show how to use the templates in the Galerkin integral equation method underlining some numerical implementation issues. Finally in Section 5 we present several example results on typical IC, package 
and PCB simple interconnect structures.

\section{BACKGROUND}

\subsection{The Mixed Potential Integral Equation For- mulation (MPIE)}

As in $[6,17,14]$ the following set of integral equations can be used for the solution of the conductor current distribution, $\mathbf{J}$, and of the conductor surface charge, $\rho$,

$$
\begin{gathered}
\frac{\mathbf{J}(\mathbf{r})}{\sigma}+j \omega \frac{\mu}{4 \pi} \int_{V} \mathbf{J}\left(\mathbf{r}^{\prime}\right) \frac{e^{j \frac{\omega}{c}\left|\mathbf{r}-\mathbf{r}^{\prime}\right|}}{\left|\mathbf{r}-\mathbf{r}^{\prime}\right|} d \mathbf{r}^{\prime}=-\nabla \phi, \\
\frac{1}{4 \pi \varepsilon} \int_{S} \rho\left(\mathbf{r}^{\prime}\right) \frac{e^{j \frac{\omega}{c}\left|\mathbf{r}-\mathbf{r}^{\prime}\right|}}{\left|\mathbf{r}-\mathbf{r}^{\prime}\right|} d \mathbf{r}^{\prime}=\phi(\mathbf{r}), \\
\nabla \cdot \mathbf{J}(\mathbf{r})=0, \\
\hat{\mathbf{n}} \cdot \mathbf{J}(\mathbf{r})=j \omega \rho(\mathbf{r}),
\end{gathered}
$$

where $V$ and $S$ are the union of the conductor volumes and surfaces, $\phi$ is the scalar potential on the conductor surfaces, $\mu$ is the magnetic permeability, $\varepsilon$ is the dielectric constant in free space, $\sigma$ is the conductors' conductivity, $\omega$ is the angular frequency of the conductor excitation and $c$ is the speed of light.

\subsection{Discretization and Galerkin procedure}

In order to solve (1)-(4) numerically, it is common to introduce an approximate representation of the volume currents and surface charges as a weighted sum of a finite set of basis functions $\mathbf{w}_{j} \in C^{3}$ and $v_{m} \in C^{1}$ as in

$$
\begin{aligned}
& \mathbf{J}(\mathbf{r}) \approx \sum_{j} \mathbf{w}_{j}(\mathbf{r}) I_{j} \\
& \rho(\mathbf{r}) \approx \sum_{m} v_{m}(\mathbf{r}) q_{m},
\end{aligned}
$$

where $I_{j}$ and $q_{m}$ are the basis function weights.

According to the Galerkin procedure [16], one can substitute the representations (5) and (6) into (1) and (2), and then require that the equation residuals are orthogonal to the basis functions. In this way one generates a system of equations for the weights,

$$
\begin{aligned}
&\left\langle\frac{\sum_{j} \mathbf{w}_{j}(\mathbf{r}) I_{j}}{\sigma}+\frac{j \omega \mu}{4 \pi} \int_{V} \sum_{j} \mathbf{w}_{j}\left(\mathbf{r}^{\prime}\right) I_{j} \frac{e^{j \frac{\omega}{c}\left|\mathbf{r}-\mathbf{r}^{\prime}\right|}}{\left|\mathbf{r}-\mathbf{r}^{\prime}\right|} d \mathbf{r}^{\prime}+\nabla \phi, \mathbf{w}_{\mathbf{i}}\right\rangle=0 \\
&\left\langle\frac{1}{4 \pi \varepsilon} \int_{S} \sum_{m} v_{m}\left(\mathbf{r}^{\prime}\right) q_{m} \frac{e^{j \frac{\omega}{c}\left|\mathbf{r}-\mathbf{r}^{\prime}\right|}}{\left|\mathbf{r}-\mathbf{r}^{\prime}\right|} d \mathbf{r}^{\prime}-\phi(\mathbf{r}), v_{l}\right\rangle=0
\end{aligned}
$$

where the inner products are defined as

$$
\begin{aligned}
& \left\langle\mathbf{f}(\mathbf{r}), \mathbf{w}_{\mathbf{i}}(\mathbf{r})\right\rangle=\int_{V} \mathbf{w}_{\mathbf{i}}{ }^{*}(\mathbf{r}) \cdot \mathbf{f}(\mathbf{r}) d \mathbf{r} \\
& \left\langle g(\mathbf{r}), v_{l}(\mathbf{r})\right\rangle=\int_{S} v_{l}^{*}(\mathbf{r}) g(\mathbf{r}) d \mathbf{r} .
\end{aligned}
$$

As in [17], a matrix equation is obtained of the form

$$
\left[\begin{array}{cc}
R+j \omega L & 0 \\
0 & P
\end{array}\right]\left[\begin{array}{l}
I \\
q
\end{array}\right]=\left[\begin{array}{l}
V \\
\phi
\end{array}\right]
$$

where $I$ and $q$ are vectors of current and charge basis function weights, respectively, and $\phi$ and $V$ are the vectors generated by inner products of the surface potential or the volume potential gradient with the basis functions. From the Galerkin condition, using the same notation as for instance in [14] the matrices $R, L$ and $P$ are

$$
\begin{aligned}
R_{i j} & =\frac{1}{\sigma} \int_{V} \mathbf{w}_{i}^{*}(\mathbf{r}) \cdot \mathbf{w}_{j}(\mathbf{r}) d \mathbf{r} \\
L_{i j} & =\frac{\mu}{4 \pi} \int_{V} \int_{V} \mathbf{w}_{i}^{*}(\mathbf{r}) \cdot \mathbf{w}_{j}\left(\mathbf{r}^{\prime}\right) \frac{e^{j \frac{w}{c}\left|\mathbf{r}-\mathbf{r}^{\prime}\right|}}{\left|\mathbf{r}-\mathbf{r}^{\prime}\right|} d \mathbf{r}^{\prime} d \mathbf{r} \\
P_{l m} & =\frac{1}{4 \pi \varepsilon} \int_{S} \int_{S} v_{l}^{*}(\mathbf{r}) v_{m}\left(\mathbf{r}^{\prime}\right) \frac{e^{j \frac{w}{c}\left|\mathbf{r}-\mathbf{r}^{\prime}\right|}}{\left|\mathbf{r}-\mathbf{r}^{\prime}\right|} d \mathbf{r}^{\prime} d \mathbf{r} .
\end{aligned}
$$

Equation (9) can be combined with the remaining equations (3) and (4) in several ways $[6,17,18]$, to generate a dense system of equations for the basis function weights. Iterative methods combined with fast matrix-vector product techniques are then commonly used to solve such system $[1,2,3,4,5]$.

\subsection{The classical piece-wise constant basis func- tions approach}

A classical choice for the basis functions is to use piece-wise constant functions. For instance small panels with a uniform charge distribution can be used for the surface charge [1]. Short thin filaments with a uniform current distribution can be used for the volume currents $[6,7,2]$. The current basis functions are generated by first subdividing long wires into a large number of sections that are short compared to the wavelength of the highest frequency of interest. The interiors of each conductor section is then divided into a bundle of parallel thin filaments.

Most fast methods for solving integral equations are based on approximating distant interactions. Such methods can not be used to reduce the cost of computing the interaction between the thin filaments in each conductor section, as these filaments are not distant from each other. The interactions between filaments in a section must be resolved "directly", and it then follows that the cost of using many filaments for each section grows as the square of the number of filaments per section. Therefore, finding a different basis which uses fewer functions to represent current distribution in each conductor section is an important efficiency consideration, even when using fast solver.

\section{PRE-COMPUTATION OF THE PROXIM- ITY TEMPLATE BASIS FUNCTIONS}

In this Section, we describe our procedure to construct a set of template basis functions for the discretization of the conductor volumes within the context of an integral equation electromagnetic field solver. As in the classical piece-wise constant approach [6, $7,2]$ described in Section 2.3, or as in the conduction modes approach $[14,15]$, we assume that the current flows only along the length of the conductors, and that long conductors are subdivided into sections short compared to the smallest wavelength of interest. We then categorize and label each conductor section according to its cross-section "type". Each "type" is uniquely identified by its cross-section dimensions and shape. For instance, for wires with a trapezoidal cross-section: width, thickness and etching slope could be used as identifying parameters.

Often when performing an electromagnetic analysis, one is interested in the current (or fields) distribution at a particular excitation frequency, or in the impedance at some terminals for several excitation frequencies. For each frequency of interest and for each wire cross-section "type", we pre-compute off-line a set of proximity template basis functions. Each basis function is constructed by solving a small simulation experiment: 

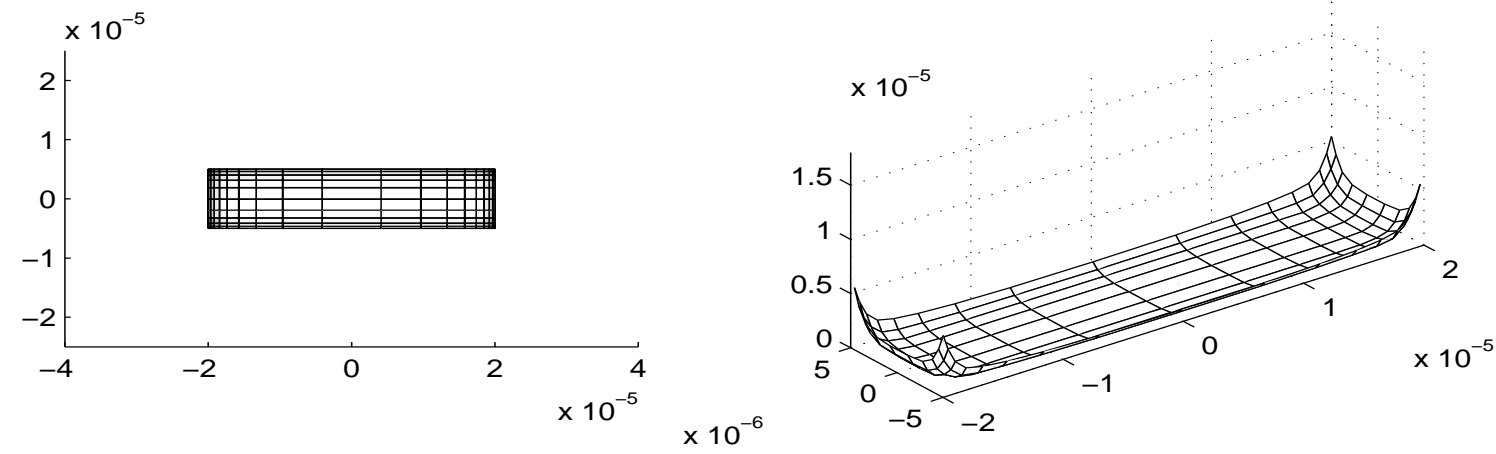

Figure 1: Example of the first proximity template basis function. On the left, we show a cross-section of the simulation experiment setup used to pre-compute the basis function. The basis function is defined as the current density (shown on the right) resulting on the cross-section of the wire.

1. Given a cross-section type, for the construction of the first template basis function we consider one wire not interacting with any other wire, and excited with a unity current source at the frequency of interest. For the solution of this simple problem we use a very fine piece-wise constant thin filament discretization method $[6,7,2]$. We then choose as basis function the current density profile derived on the entire cross-section by this analysis. We show on the left of Fig. 1 the thin filament discretization of the wire cross-section and to its right the resulting cross-section current density that we use as first basis function. In Fig. 1 the wire cross-section is rectangular, but in general cross-section shapes can be handled in the same way by our procedure. From an intuitive point of view, the template basis function described here is used to capture skin effect phenomena.

2. Other basis functions are then constructed to capture proximity effect phenomena. In order to capture proximity effect phenomena due to wires on the side, we construct a second template basis function by solving a second simple experiment. In this second experiment we consider two wires not interacting with any other wire. The cross-section of the "main" wire is chosen equal to the cross-section shape and dimensions for the "type" under consideration. A second auxiliary wire is located on one of the two sides of the main wire, as close to the "main" wire as the technology fabrication process would allow. The auxiliary wire is chosen with the minimum width and thickness allowed by the technology fabrication process. Fig. 2 shows on the left the cross-section configuration of the two wires. For the analysis of this problem we use a classical and very fine piece-wise constant discretization for both such wires. We short them together on one side, apply a unity current source at the remaining two terminals, and solve for the current density within the conductors. We finally define as second proximity template basis function the current density profile observed on the main wire. On the right in Fig. 2 we show the cross-sectional current density of the second basis function.

3. We proceed constructing additional proximity template basis functions using the procedure described in point 2 above, but every time moving the auxiliary wire in a different location around the main wire, always as close to the main wire as the technology fabrication process allows. Fig. 3 shows other two examples of template basis functions with their corresponding experiment setups for the same cross-section as in Fig. 1 and 2.

\subsection{Choosing the number of template basis functions per wire cross-section}

More specifically, the total number of template basic functions precomputed for each cross-section type can be decided according to the following considerations.

In some cases, one only needs to use a total of three proximity templates for each cross-section type: a "skin effect template" constructed as in Fig. 1, and two "side proximity templates", one for the right side as in Fig. 2 and one for the left side (typically symmetric to the one in Fig. 2). This choice is typically appropriate for wires on most Printed Circuit Board (PCB) applications, where the separation between different layers is particularly large, and proximity effects are only observed in correspondence of "side by side" wires, and not in correspondence of wires on different layers.

When separation between metalization layers is small as in packages and in integrated circuits, one needs to be able to account for proximity effects due not only to wires "side by side" but also due to wires on upper and lower layers. In this case, for thin wires we use a total of nine templates: a "skin effect template", four proximity templates constructed using an auxiliary wire moved to each of the four sides of the main wire, and four proximity templates with the auxiliary wire moved to each of the corners around the main wire. Fig. 1, 2, and 3, show four out of nine of such templates. If appropriate, symmetry of the cross-section can be exploited to infer the other five template results.

Finally, in the case of considerably wide wires, in addition to the nine templates previously described, one needs to use a few more proximity templates to capture appropriately proximity effects due to thin wires in any location above or below such wide wire. In our implementation we precompute templates using an auxiliary wire that for each template is moved in different locations around the main wire. We remind the reader that the auxiliary wire width an thickness are chosen as the minimum allowed by the technology process, and that the auxiliary wire is located at the minimum distance from the main wire allowed by the technology process. For each template we then move the auxiliary wire to locations each separated by 4 times the auxiliary wire width.

\subsection{Accuracy and basis function reachness}



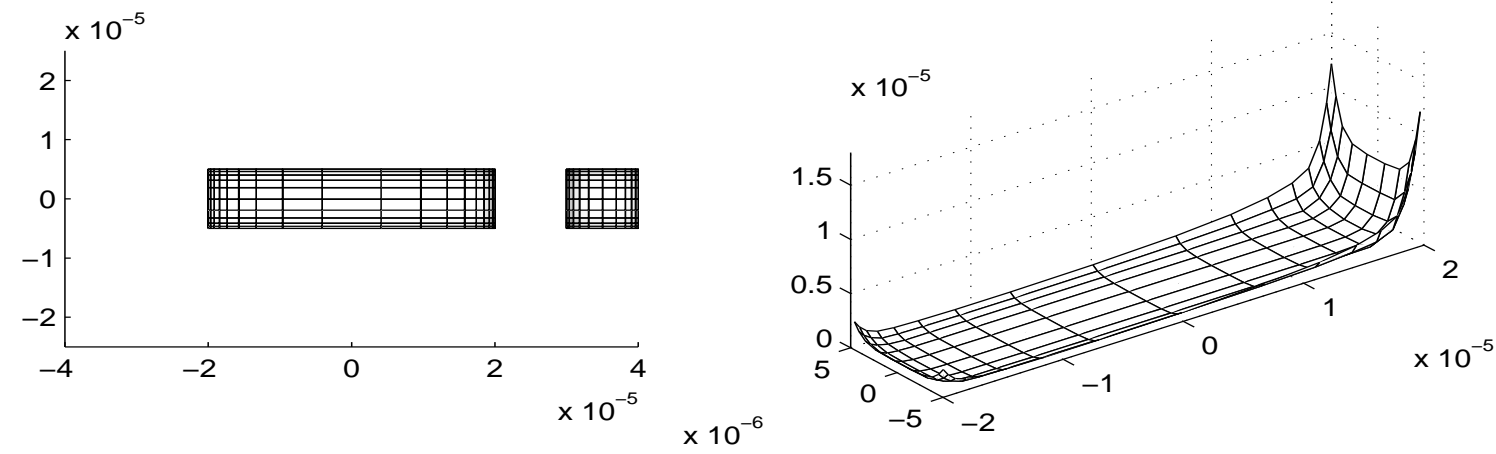

Figure 2: Example of the second proximity template basis function. On the left, a cross-section of the simulation experiment setup used to pre-compute the basis function. On the right the basis function itself: i.e. the current density resulting on the main wire cross-section.

The accuracy of the final solution is related to the ability of the chosen basis functions to "explain" most of the cross-sectional current density capturing current crowing in different parts of each cross-section due to the specific locations of nearby wires. More precisely, in linear algebra terms: when considering the cross-sectional current density as a vector, the accuracy of the final solution is related to the ability of the chosen basis functions to "span" most of the subspace generated by all practical current density vectors. In general the accuracy of the final solution can be arbitrarily improved if the set of all basis functions that one can choose from is sufficiently reach to span the entire subspace of all practical solutions. In our case, in theory the basis function set is quite reach since one could increase the accuracy of the final solution by simply adding more and more basis functions, one for each possible practical location of nearby wires. However we have observed experimentally (see Examples 5.2 and Example 5.1) that the procedure in 3 and 3.1 constructs a much smaller set of basis functions at the same time still allowing of a good final solution accuracy.

\subsection{Advantages and disadvantages of the prox- imity template basis functions}

From the construction procedure described above in 3 and 3.1 one can notice several advantages and disadvantages of our template basis functions in particular when compared to other higher order basis function choices such as the "conduction modes" described in $[14,15]$. Among the advantages it can be noticed that:

- our template basis functions can handle any wire cross-section shape, i.e. the common trapezoidal cross-sections due to chemical etching slopes. In the conduction modes basis function approach $[14,15]$, instead, only cross-section shapes for which analytic solutions of the diffusion equation are available can be handled, i.e. mainly rectangular and cylindrical cross-sections.

- our template basis functions can capture proximity effects due to thin wires above very wide wires (as shown later in Example 5.2 and Fig. 5) that are not captured by the "conduction mode" approach.

Among the disadvantages of our template basis functions, we remind the reader that:

- a complete set of template basis functions need to be precomputed for each wire cross-section "type" (i.e. shape and dimensions). Fortunately, one can further observe that often the actual number of wire cross-section types on a typical PCB, package or IC is quite limited. For instance the etching slope can be assumed constant for all cross-sections for a given process. The variability of the wire thickness is limited to the number of metalization layers in the design. Also the variability of the wire width parameter in practical designs is often limited to a finite and small set of admissible values by design rules or CAD tools. It is also worth noticing that once the template basis functions are computed they can be stored in a file and re-used for subsequent designs based on the same process technology.

- Another disadvantage of our proximity templates compared to the conduction modes is that a complete set of template basis functions need to be pre-computed for each frequency of interest. Typically one is not interested in a large number of frequencies. For instance in digital interconnect one is typically only interested in the clock frequency and its first 10 to 15 harmonics. Once again, one can further notice that once the template functions are calculated for a particular frequency, they can be stored and re-used in subsequent designs for analysis at that same frequency. However admittedly a significant advantage of the conduction mode basis functions over our proximity templates is the availability of the conduction modes in analytical form which can be exploited when performing model order reduction.

\subsection{Representation of basis functions}

As just observed in the previous Section, we represent our basis functions with a piecewise constant values of the current density on each small cross-sectional filament. In this way for each basis function we only need to store some information on the discretization scheme from which one can easily derive filament geometries (e.g. width of corner filament and incremental ratio between nearby filaments), and a vector with the values of current density on each filament.

One could think of using more efficient representations in terms of some interpolation functions in order to save some storage memory and some computation time in the Galerkin integral computations. We expect however to obtain the most advantage by fitting our templates basis functions to interpolation function not only to represent their shape along the wire cross-section but also and above all to capture their dependency from frequency. In fact, this 

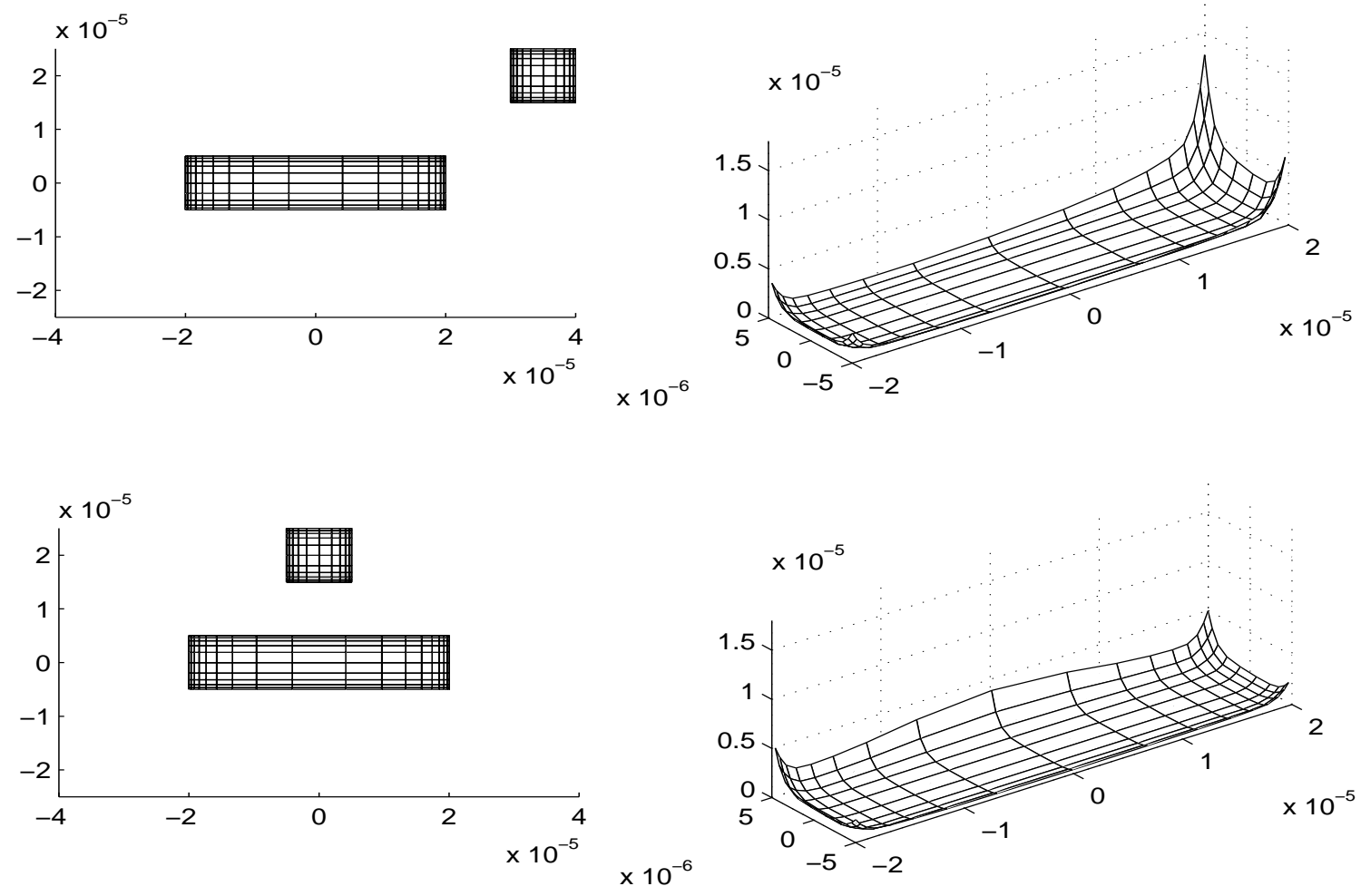

Figure 3: Example of other two proximity template basis function. On the left, a cross-section of the simulation experiment setups used to pre-compute the basis functions. On the right the basis functions themselves: i.e. the current densities resulting on the main wire cross-sections.

could allow us to perform model order reduction with our template basis functions as efficiently as with the conduction modes. However, we have not verified yet the practical feasibility of such procedure.

\section{PARASITIC EXTRACTION FOR A LARGE COLLECTION OF INTERCONNECT}

Given a large collection of wires, for a given frequency of interest, each wire is associated with the set of pre-computed proximity template basis functions corresponding to its cross-section type. The basis functions chosen in this way, together with a standard Galerkin procedure [16], are used to discretize the Mixed Potential Integral Equation (MPIE) and calculate the overall resistance $R$ and the partial inductance $L$ matrices in eq. (10) and (11) as shown in Section 2.2. Accumulation of charge on the surfaces of the conductors can still be handled for example using the classical piecewise constant discretization of such surface into small panels as described in Section 2.3. A mesh analysis technique [17] is then finally used to set up a linear system of equations that can be solved to find the weights $\mathbf{w}_{j}$ and $\mathbf{v}_{m}$ associated with each single basis function.

From a numerical implementation prospective one can observe that the proximity template basis functions as constructed in Section 3 are not orthogonal. The resistance matrix for instance is block diagonal. In general, when the basis functions are almost linearly dependent, their associated coefficients representing the final solution may result very large, similar in magnitude, and possibly of opposite phases partially canceling each others, which may produce errors when using a finite precision representation. One can avoid this problem and achieve better numerical stability by ortho-normalizing the basis functions before using them with for instance a "Modified Gramm-Schmidt" procedure [19]. Another advantage of orthonormalizing the basis functions is that a completely diagonal resistance matrix is produced, which is convenient for instance when performing a subsequent model order reduction step that may require an inversion of such matrix. The orthogonalization procedure is quite fast and most importantly it is part of the "precomputation" phase, hence it does not affect the speed and memory performance during the analysis of a very large collection of interconnect.

As a final remark, it can be noticed that our proximity templates basis functions can be used in combination with fast matrix solvers $[1,2,3,4,5]$.

\section{EXAMPLES}

\subsection{Capturing proximity effect between two wires at arbitrary distance}

In this Section, we intend to show with an example that although our proximity templates are constructed using an auxiliary wire very close to the main wire, such template basis functions can successfully capture proximity effects due to wires at any arbitrary distance. Let us consider for instance a typical PCB wire $250 \mu \mathrm{m}$ wide, and $35 \mu \mathrm{m}$ thick. In this example we used a set of three template basis functions per cross-section. One template was constructed using one wire alone with a $250 \mu \mathrm{m}$ x $35 \mu \mathrm{m}$ cross- 

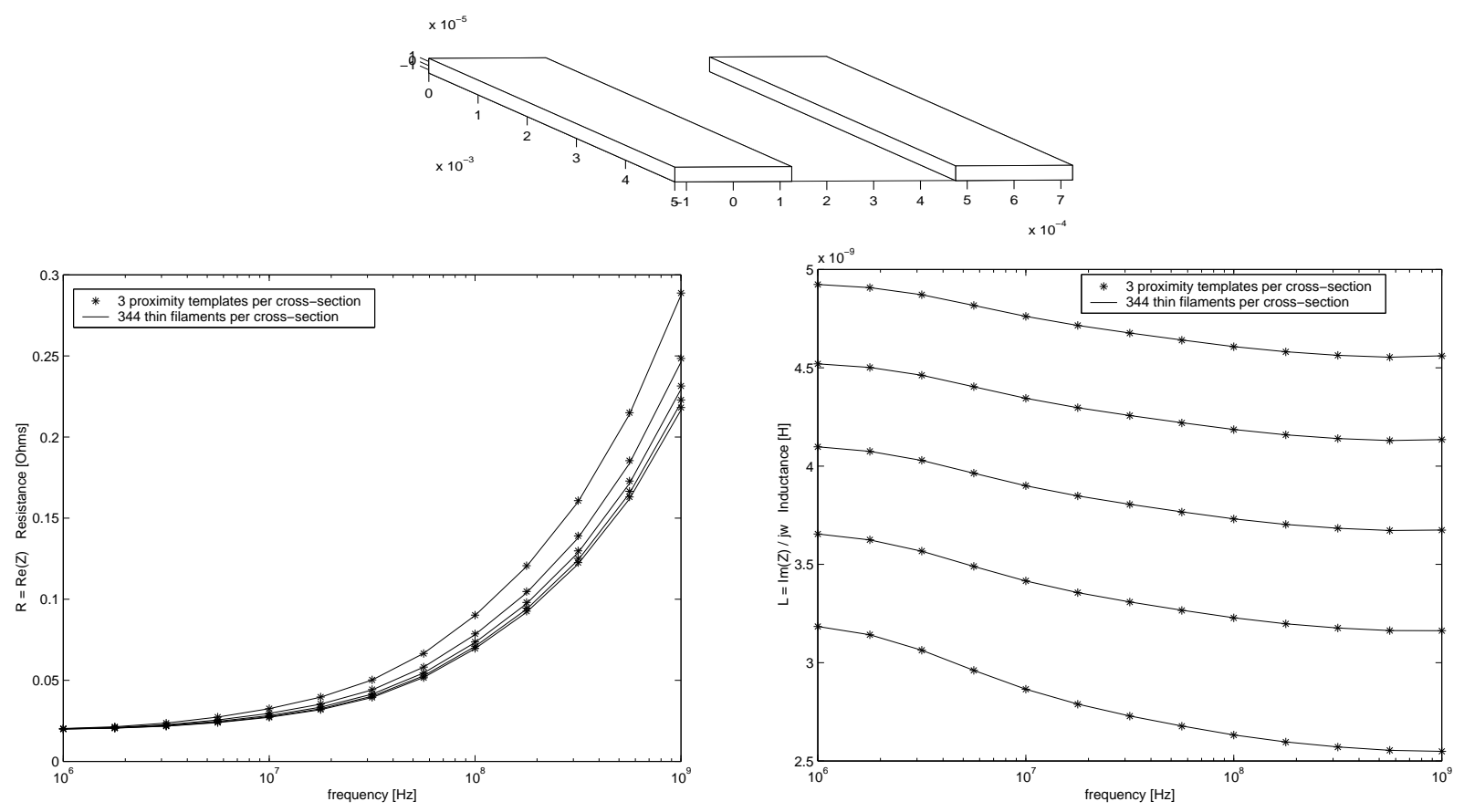

Figure 4: Frequency response of two shorted PCB wires: real part of the impedance on the left, and imaginary part divided by $\omega$ on the right. The different curves represent different distances between the two wires: $100 \mu \mathrm{m}, 190 \mu \mathrm{m}, 305 \mu \mathrm{m}, 448 \mu \mathrm{m}$, and $629 \mu \mathrm{m}$ (from top to bottom on the left and from bottom to top on the right). The continuous lines are the results obtained using the classical thin filament method. The small crosses are the results obtained using three template basis functions pre-computed for a minimum separation distance $100 \mu \mathrm{m}$.

section. A second template was constructed using one main wire (cross-section: $250 \mu \mathrm{m} \times 35 \mu \mathrm{m}$ ) in the center and one auxiliary wire $(250 \mu \mathrm{m} \times 35 \mu \mathrm{m})$ on one side at a separation distance of $100 \mu \mathrm{m}$. A third template was constructed by moving the auxiliary wire to the other side at the same separation distance. For the construction of the basis functions, we discretized each wire into $24 \times 14=344$ thin filaments. After the three template basis functions have been constructed, we used them in the integral equation Galerkin procedure described in Section 2.2 to calculate the frequency response of two wires with the same cross-section at different separation distances: $100 \mu \mathrm{m}, 190 \mu \mathrm{m}, 305 \mu \mathrm{m}, 448 \mu \mathrm{m}$, and $629 \mu \mathrm{m}$. We compare in Fig. 4 the result obtained using our three proximity template basis functions per cross-section with the result obtained using 344 thin filaments basis functions per cross section. Of course one can expect a negligible error when the wires' separation is exactly equal to the separation used for the construction of the basis functions $(100 \mu \mathrm{m})$. However, we also observed an equally very small error (worst case $0.7 \%$ error for the real part of the impedance, and a $0.01 \%$ error for the imaginary part divided by $\omega$ ) for the case in which the separation between the two wires increased to an arbitrary distance and did not coincide anymore with the separation used during the construction of the basis functions.

\subsection{Capturing proximity effect between a thin wire in an arbitrary location above a wide wire}

From the previous example we have seen that the proximity templates is an approach at least as efficient as the conduction modes approach [14] in terms of used number of unknowns. In addition, we show in this example that the proximity templates can successfully capture one particular case not captured by the conduction modes approach: proximity effects between a thin wire above and close to a very wide wire. Let us consider for instance a package wire $40 \mu \mathrm{m}$ wide and $10 \mu \mathrm{m}$ thick. Let us pre-compute a set of nine proximity effects basis functions for this wire. Fig. 1, 2, and 3 show four of such nine basis functions for the cross-section type described in this example. The auxiliary wire is $10 \mu \mathrm{m}$ wide and is moved into several locations all around the main wire all at a distance of $10 \mu \mathrm{m}$. After the computation of the basis functions, we have setup the experiment on top of Fig. 5. The small wire is $10 \mu \mathrm{m}$ $\mathrm{x} 10 \mu \mathrm{m}$, the wider wire right below it is $40 \mu \mathrm{m} \times 10 \mu \mathrm{m}$ at a $10 \mu \mathrm{m}$ separation. We can also notice that the small wire is off center by $4 \mu \mathrm{m}$ so that its location does not coincide with one of the locations used for the basis function construction (compare the cross-section in Fig. 3 with the cross-section of the geometry in Fig. 5). The two remaining pictures in Fig. 5 compare the cross sectional current density resulting from using our set of nine pre-computed proximity template basis functions (left), with the result (on the right) obtained using a set of $16 \times 9=144$ thin filaments basis functions. We conclude that the proximity templates provide accurate results not only for wires at an arbitrary distance as shown in Example 5.1, but also for wires located "in between" the original locations used for the basis functions construction.

\subsection{A package power and ground distribution example}

Finally we show here a package power and ground distribution grid Example (Fig. 6). Wires are $10 \mu \mathrm{m}$ wide and $5 \mu \mathrm{m}$ thick. Vertical separation between layers is $5 \mu \mathrm{m}$. Side separation between Gnd and Vdd lines is $1 \mathrm{~mm}$. The total package size is $12 \mathrm{~mm} \times 12 \mathrm{~mm}$. We assumed bond wires connections shorting Gnd and Vdd wires to an underneath PCB on all 4 corners of the package. We can no- 

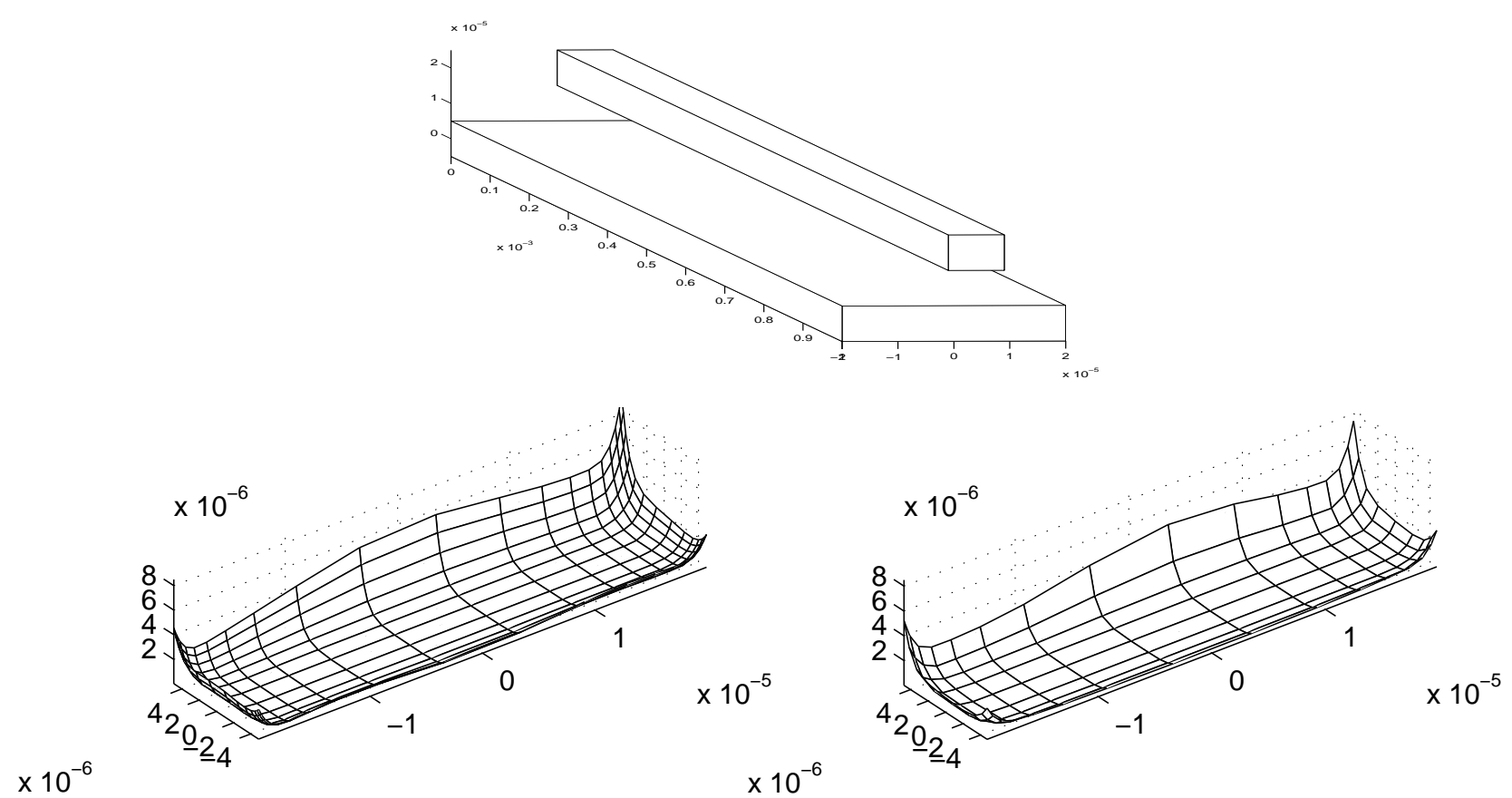

Figure 5: Package trace cross sectional current density "reconstructed" from a set of nine pre-computed proximity template basis functions (picture on the left), compared to the current density (picture on the right) from using a set of $16 \times 9=144$ thin filaments basis functions. A larger current distribution can be noticed on one edge and on one corner of the cross-section due to the proximity of the small wire. Note that the location of the small wire is off center, hence it does not coincide with any of the locations used to pre-compute the nine template basis functions. One can notice that the current density is still captured accurately.

tice that only one type of cross-section is present in this design. For that cross-section type, we have pre-computed a set of three basis functions as in Example 5.1. We have then used our three basis functions per segment to discretize the entire geometry and find the frequency response at one particular node of the grid: the node at $\mathrm{x}=4 \mathrm{~mm}$ and $\mathrm{y}=0 \mathrm{~mm}$ (see Fig. 6). In our simulations we have also included the effects of charge accumulation on the surface of the conductors using a piece-wise constant discretization into small panels. In Fig. 6 we compare the frequency response of the grid at the node indicated above according to our three proximity templates per wire versus the frequency response obtained using a thin filament discretization with $5 \times 4=20$ thin filaments per each wire segment of the grid. Our approach required a total of $48 \times 3=144$ unknowns for the conductor currents, while to get a similar accuracy with the thin filament approach we had to use a total of $48 \times 20=960$ unknowns. In particular, for our proximity templates approach we observed from the admittance phase vs. frequency curve in Fig. 6 a worst case error of $0.5 \%$ in the position of the resonances. We observed from the admittance amplitude vs. frequency curve a worst case $7 \%$ error in amplitude at the resonances, where the impedance is mainly determined by skin effects and proximity effects.

\section{CONCLUSIONS}

In this paper we have described a procedure to construct a set of template basis functions for the discretization of conductor volumes in an integral equation method. The template basis functions are pre-computed off-line using small simulation experiments. The templates can capture successfully both skin effect and proximity effects. Our examples show that compared to the thin filament methods they provide the same 7 to 20 improvement factors in terms of number of unknowns reported by the conduction modes approach [14]. In addition the proximity templates can be employed in applications with wire cross-sections of arbitrary shape, and with proximity effects on wide wires due to above and close thin wires.

\section{ACKNOWLEDGMENTS}

The authors would like to thank Dr. Joel Phillips (with Cadence Berkeley Labs) for useful feedback and discussion.

\section{REFERENCES}

[1] K. Nabors and J. White. Fastcap: a multipole accelerated 3-d capacitance extraction program. IEEE Trans. on Computer-Aided Design of Integrated Circuits and Systems, 10(11):1447-59, November 1991.

[2] M. Kamon, M. J. Tsuk, and J. K. White. Fasthenry: A multipole-accelerated 3-d inductance extraction program. IEEE Trans. on Microwave Theory and Techniques, 42(9):1750-8, September 1994.

[3] J. R. Phillips and J. White. A precorrected-fft method for electrostatic analysis of complicated 3-d structures. IEEE Trans. on Computer-Aided Design of Integrated Circuits and Systems, 16(10):1059-1072, October 1997.

[4] J. Tausch and J. White. A multiscale method for fast capacitance extraction. In Design Automation Conference, pages 537-42, June 1999.

[5] S. Kapur and D. Long. Large scale capacitance calculations. In Design Automation Conference, pages 744-9, June 2000.

[6] A. E. Ruehli. Equivalent circuit models for three dimensional 

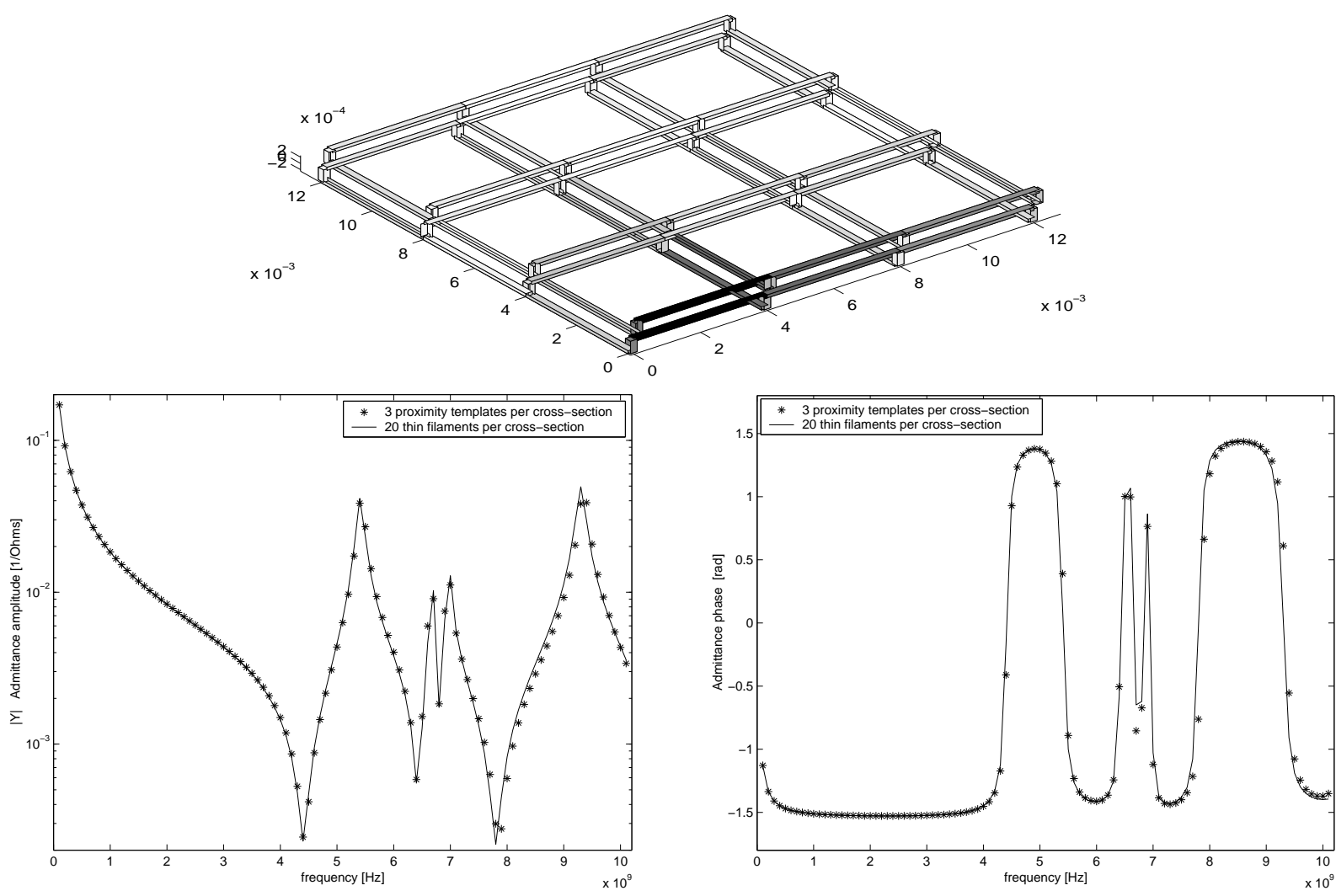

Figure 6: A simplified representation of a power and ground grid of a package or of an integrated circuit (picture on top). Gray shading on the the grid indicate current distributions calculated by our solver when a current source excites the grid node $x=4 \mathrm{~mm}$ and $y=0 \mathrm{~mm}$. On the left we show the admittance amplitude, on the right the admittance phase vs. frequency observed at that same grid node. In this example we used three proximity template basis functions for each wire cross-section.

multiconductor systems. IEEE Trans. on Microwave Theory and Techniques, 22:216-221, March 1974.

[7] W. T. Weeks, L. L. Wu, M. F. McAllister, and A. Singh. Resistive and inductive skin effect in rectangular conductors. IBM J. Res. Develop., 23(6):652-660, November 1979.

[8] P. Silvester. Modal network theory of skin effect in flat conductors. Proc. IEEE, 54(9):1147-1151, September 1966.

[9] M. J. Tsuk and J. A. Kong. A hybrid method for the calculation of the resistance and inductance of transmission lines with arbitrary cross section. IEEE Trans. on Microwave Theory and Techniques, 39(8):1338-1347, August 1991.

[10] E. Tuncer, B. T. Lee, and D. P. Neikirk. Interconnect series impedance determination using a surface ribbon method. IEEE Topical Meeting on Electrical Performance of Electronic Packaging, pages 249-252, November 1994.

[11] K. M. Coperich, A. E. Ruehli, and A. Cangellaris. Enhanced skin effect for partial-element equivalent-circuit (peec) models. IEEE Trans. on Microwave Theory and Techniques, 48(9):1435-42, September 2000.

[12] J. Wang, J. Tausch, and J. White. A wide frequency range surface integral formulation for 3-d rlc extraction. In IEEE/ACM Internat. Conf. on Computer-Aided Design, pages 453-7, 1999.

[13] Z. Zhu, J. Huang, B. Song, and J. White. Improving the robustness of a surface integral formulation for wideband impedance extraction of $3 \mathrm{~d}$ structures. In IEEE/ACM
Internat. Conf. on Computer-Aided Design, pages 592-7, 2001.

[14] L. Daniel, A. Sangiovanni-Vincentelli, and J. White. Interconnect electromagnetic modeling using conduction modes as global basis functions. In IEEE Topical Meeting on Electrical Performance of Electronic Packaging, pages 203-206, October 2000.

[15] L. Daniel, A. Sangiovanni-Vincentelli, and J. White. Using conduction modes basis functions for efficient electromagnetic analysis of on-chip and off-chip interconnect. In Design Automation Conference, June 2001.

[16] R. F. Harrington. Field Computation by Moment Methods. MacMillan, 1968.

[17] M. Kamon, N. Marques, and J. White. Fastpep: a fast parasitic extraction program for complex three-dimensional geometries. In IEEE/ACM Internat. Conf. on Computer-Aided Design, pages 456-460, November 1997

[18] N. Marques, M. Kamon, J. White, and L. M. Silveira. A mixed nodal-mesh formulation for efficient extraction and passive reduced-order modeling of $3 \mathrm{~d}$ interconnects. In Design Automation Conference, pages 297-302, June 1998.

[19] James W. Demmel. Applied Numerical Linear Algebra. SIAM Society for Industrial and Applied Mathematics, 1997. 